\title{
Personalized Recommendation Algorithm for Web Pages Based on Associ ation Rule Mining
}

\author{
Lu Xing-Hua ${ }^{1, *}$, Ye Wen-Quan ${ }^{1}$, and Liu Ming-Yuan ${ }^{1}$ \\ ${ }^{1}$ Huali College Guangdong University of Technology, 511325 Guangdong Guangzhou, China
}

\begin{abstract}
In order to improve the user ' $s$ ability to access websites and web pages, according to the interest preference of the user, the personalized recommendation design is carried out, and the personalized recommendation model for web page visit is established to meet the personalized interest demand of the user to browse the web page. A webpage personalized recommendation algorithm based on association rule mining is proposed. Based on the semantic features of web pages, user browsing behavior is calculated by similarity computation, and web crawler algorithm is constructed to extract the semantic features of web pages. The autocorrelation matching method is used to match the features of web page and user browsing behavior, and the association rules feature quantity of user browsing website behavior is mined. According to the semantic relevance and semantic information of web users to search words, fuzzy registration is taken, Web personalized recommendation is obtained to meet the needs of the users browse the web. The simulation results show that the method is accurate and user satisfaction is higher.
\end{abstract}

\section{INTRODUCTION}

In today's big data network and information age, the scale of network information is expanding. In comprehensive browsers and websites, people need to excavate web pages that meet their personalized needs from massive network information and browse information. The information that the users care about are extracted from the massive network data to improve the efficiency of the user's web browsing. In the current network environment, the flow of data and information is very huge, Internet users have put forward a higher request for the accuracy of network search, everyone has different content and hobby to the web page[1]. It needs to provide personalized customization requirements based on user preferences. According to users' interests and needs of work and life, we recommend website information recommendation and build user project personalized recommendation model. The research of related webpage recommendation algorithm is of great significance in improving user browsing efficiency and satisfaction.

The recommended model is established in the construction of trust relationship and semantic information model of web user mining based on Web personalization, the web user's prior information browsing and assess the needs of the development of personalized information recommendation, semantic similarity, feature extraction in web pages, considering the structure and distribution of Web site users demand, develop the project of personalized service information push and Web Recommendation, experience and lay the foundation for more efficient[2]. At present, the typical personalized recommendation methods are mainly based on Twittomender system recommendation model, TF-IDF keyword weight measurement personalized recommendation model and semantic information mining model. By extracting semantic similarity information feature quantity and combining user's interest preference, semantic association matching is carried out to improve the recommendation accuracy of web pages. The reference [3] proposes a collaborative recommendation algorithm based on the similarity of "personalized, the trust degree of conditional probability analysis method to construct the model of personalized recommendation reliability, feature analysis and extraction of user browsing behavior are taken to realize the attribute classification browsing behavior, but the computation cost is too large, the anti-interference ability is not strong In reference [4], it proposes a recommendation model of phase space reconstruction and Web Crawler personalized based on association rules and characteristics in the reconstructed phase space to extract relevant information on the web, personalized mining is taken to improve user browsing, but this method is not good for the real-time web data mining of massive the accuracy is not high[4].

In solvinge the above problems, this paper proposes a personalized recommendation algorithm for web pages based on association rule mining. A webpage personalized recommendation algorithm based on association rule mining is proposed. Based on the semantic features of web pages, user browsing behavior is calculated by similarity computation, and web crawler algorithm is constructed to extract the semantic features 
of web pages. The autocorrelation matching method is used to match the features of web page and user browsing behavior, and the association rules feature quantity of user browsing website behavior is mined. Finally, the simulation experiment is carried out to obtain the validity conclusion.

\section{INDIVIDUAL USER BROWSING BEHAVIOR MODEL AND WEB CRAWLER ALGORITHM}

\subsection{Modeling of user browsing behavior}

$\mathrm{I}=\{\mathrm{i} 1, \mathrm{i} 2, \ldots, \mathrm{in}\}$ and $\mathrm{U}=\{\mathrm{u} 1, \mathrm{u} 2, \ldots, \mathrm{um}\}$ are used to denote the item set and user set of web browsing and website visiting respectively. The matrix $\mathrm{m} \times \mathrm{n}$ is used to represent the comprehensive evaluation set of $m$ users accessing $n$ web pages:

$$
\left(\begin{array}{ccc}
r_{u_{1}, i_{1}} & \ldots & r_{u_{1}, i_{n}} \\
\vdots & \ddots & \vdots \\
r_{u_{m}, i_{1}} & \cdots & r_{u_{m}, i_{n}}
\end{array}\right)
$$

According to the preference degree of web pages visited by users ua and ub, the similarity features between users are calculated, and the semantic attribute classification and access control of web pages are carried out by using autocorrelation matching method[6]. The interface has different expression forms according to different attributes, which affects the generation of web page lattice recommendation results. The browsing behavior attribute matching model of web pages can be divided into keyword attributes. Range attributes and classification attributes are expressed as:

(1) Keywords attribute. Minimal combination calculation of user's interest is taken, in the database access interface page, keyword attributes and logical expression are:

$$
\mathrm{r} \text { like } \mathrm{s} \text { or } \mathrm{r} \subset \mathrm{s}
$$

Wherein, $r$ is Page access results, $s$ shows Keywords input sample conditions, collect pages, save pages, print pages are taken by users to achieve fuzzy matching and search through search engines to find a text set consisting of one or more keywords.

(2) Scope properties. Arbitrarily fill in two logical values on the web page query provider through the relationships between various browsing behaviors, representing a range of time or space :

$$
s_{1} \leq r \leq s_{2}
$$

Wherein, $s_{1}$ and $s_{2}$ represents two logical values, such as time, etc. If the user browses multiple web pages with higher similarity at a given time, the semantic feature is associated.

(3) Classification attribute. Classification attribute is a kind of accurate web page theory personalized recommendation attribute. It is based on a limited number of different values to access the query, such as based on the ID number, the IP number of the site, and so on. An accurate classification attribute of web browsing is obtained and an accurate query result value is returned. The query result requires that the input condition be strictly matched with the result so that the accurate return can be realized[7].

When the browsing behavior of the user is analyzed, the webpage personalized recommendation system association rule mining area is provided with a scalar time sequence which is $x(t), t=0,1, \cdots, n-1$, The semantic similarity calculation of the webpage is carried out by using the browsing times of the user as the quantitative characteristic information, and can be described as:

$$
u=\left[u_{1}, u_{2}, \cdots, u_{N}\right] \in R^{m N}
$$

The maximum likelihood estimation (MLE) method is used to calibrate the data stream mining region for association rule features. Combing with fuzzy inference rules, periodic estimation of user number, the maximum gradient is obtained, personalized recommendation results output difference is:

$$
A V G_{\mathrm{X}}=\frac{1}{m \times n} \sum_{x=1}^{n} \sum_{y=1}^{m}\left|G_{\mathrm{X}}(x, y)\right|
$$

Wherein, $G_{\mathrm{X}}(x, y)$ is the user's interest in the page they visit, $m, n$ are the similarity sets of webpage personalized recommendation output, and the update proportion of page browsing times is calculated. The confidence level of user's browsing behavior is obtained by using autocorrelation fusion estimation method:

$$
\rho_{X Y}=\frac{\operatorname{Cov}(X, Y)}{\sqrt{D(X)} \sqrt{D(y)}}
$$

Under the hybrid cloud framework, the distribution nodes of the data information flow association rules are given as $G_{1}=\left(M_{1}^{\alpha}{ }_{1} M^{\beta}{ }_{1}, Y_{1}\right), \quad G_{2}=\left(M^{\alpha}{ }_{2}, M^{\beta}{ }_{2}, Y_{2}\right)$, According to the frequency of web browsing in the last cycle and the current cycle, the user browsing behavior modeling is carried out, which provides the model foundation for personalized recommendation[8].

\subsection{Web crawler algorithm}

It is assumed that the web distribution is directed to the edges in the figure, in a personalized recommendation system, set $\left\{u_{1}, \ldots, u_{N}\right\}$ is a set of user nodes comprising of element nodes, $\quad\left\{v_{1}, \ldots, v_{M}\right\}$ represents a collection of untrusted nodes, $R=\left[R_{u, v}\right]_{N \times M}$ is a rating matrix for a user to a project, wherein $R_{u, v}$ is attribute interest evaluation. According to the semantic features of the similarity of user browsing behavior calculation:

$$
\begin{gathered}
\operatorname{WebJaccard}(X, Y)=\frac{P(X \cap Y)}{P(X)+P(Y)-P(X \cap Y)} \\
\operatorname{WebOverlap}(X, Y)=\frac{P(X \cap Y)}{\min (P(X), P(Y))} \\
\operatorname{WebDice}(X, Y)=\frac{2 P(X \cap Y)}{P(X)+P(Y)}
\end{gathered}
$$


Wherein, $P(X), P(Y)$ represent the probability density function for the distribution of association rules in a personalized recommendation system on a web page, $\mathrm{X}, \mathrm{Y}$ are load of data transmission, $P(X \cap Y)$ is the mutual information probability density distribution of association rule mining.

Web crawler web semantic feature extraction algorithm is designed to construct the semantic web, isomorphism ad hoc networks, node initial and residual energy network semantic concept tree is a mapping from an algebra to another algebraic structure, semantic web ad hoc network of each cluster by a cluster head, a plurality of cluster heads and cluster members, it remains the same for all related structures[9], namely mapping $\Phi: \mathrm{X} \rightarrow \mathrm{Y}$, satisfied:

$$
\Phi(x \bullet y)=\Phi(x) \circ \Phi(y)
$$

Wherein, $\bullet$ is operations on $\mathrm{X}, \circ$ is operations on $\mathrm{Y}$. In the distributed concept tree design of semantic crawler isomorphic network, the average convergence efficiency of all decision concept trees in the website is defined as the global efficiency of the network. $E(G)$ is:

$$
E(G)=\frac{1}{N(N-1)} \sum_{i \neq j} \frac{1}{d_{i j}}
$$

For the data set of data mining objects, the average path of the web crawler is calculated as follows:

$$
d_{i j}=\sum_{i^{\prime}=1}^{k} w_{i^{\prime} k} d_{i^{\prime} k}
$$

According to the above design of crawler algorithm, the semantic features of web pages are extracted, and autocorrelation matching method is used to match the features of web pages and users' browsing behavior.

\section{OPTIMIZATION OF PERSONALIZED RECOMMENDATION ALGORITHM FOR WEB BROWSING}

\subsection{Association rule feature mining}

This paper presents a personalized recommendation algorithm for web pages based on association rule mining. It is assumed that the distributed data set of web page personalization recommendation system is represented as $\mathrm{X}$, the vector set reconstructed in the finite data set phase space is:

$$
X=\left\{x_{1}, x_{2}, \cdots, x_{n}\right\} \subset R^{s}
$$

Wherein, the I feature vectors of dynamic inertial data feature points distributed in phase space $x_{i}=\left(x_{i 1}, x_{i 2}, \ldots, x_{i D}\right)$, according to similarity and neighbor set, the global optimal vector before stabilization stage is obtained as $v_{i}=\left(v_{i 1}, v_{i 2}, \ldots, v_{i D}\right)$, when making group recommendation, we cannot simply regard the group as the sum of users, we need to mine association rules, and get the difference degree of personalized recommendation association rules mining:

$$
\tilde{w}_{k}^{i}=\tilde{w}_{k-1}^{i} \frac{p\left(z_{k} / \tilde{x}_{k}^{i}\right) p\left(\tilde{x}_{k}^{i} / x_{k-1}^{i}\right)}{q\left(\tilde{x}_{k}^{i} / x_{k-1}^{i}\right)}
$$

Wherein, $\tilde{x}_{k}^{i}$ is the absolute value of the difference between every two users I of a web page, the association rule vector is:

$$
x_{i}(k+1)=x_{i}(k)+s\left(\frac{x_{j}(k)-x_{i}(k)}{\left\|x_{j}(k)-x_{i}(k)\right\|}\right)
$$

For a limited data set with $n$ samples, personalized Web page recommendation output data stream samples $x_{i}$ :

$$
x_{i}=\left(x_{i 1}, x_{i 2}, \cdots, x_{i s}\right)^{T}
$$

The method of fuzzy inference is adopted to obtain the ordered queue distribution of the feature extraction of the correlation rule:

$$
V=\left\{v_{i j} \mid i=1,2, \cdots, c, j=1,2, \cdots, s\right\}
$$

The personalized association rule characteristic mining output of the webpage browsing behavior is:

$$
U=\left\{\mu_{i k} \mid i=1,2, \cdots, c, k=1,2, \cdots, n\right\}
$$

The objective function of personalized recommendation is:

$$
J_{m}(U, V)=\sum_{k=1}^{n} \sum_{i=1}^{c} \mu_{i k}^{m}\left(d_{i k}\right)^{2}
$$

Wherein, $\left(d_{i k}\right)^{2}$ is measure distance of $x_{k}$ and $V_{i}$ sample association rules, it is:

$$
\left(d_{i k}\right)^{2}=\left\|x_{k}-V_{i}\right\|^{2}
$$

Further calculate the semantic similarity feature distribution fitness of web page personalized recommendation output:

$$
\sum_{i=1}^{c} \mu_{i k}=1, k=1,2, \cdots, n
$$

The difference perturbation is used to correct the error, and the data location mining is guided in the reconstructed phase space to improve the accuracy of web recommendation.

\subsection{Semantic relevance matching and recommendation output of Web pages}

By adopting the self - correlation matching method, the characteristics of the webpage and the browsing behavior of the user are matched, and the matching relation between the webpage recommendation and the user behavior is obtained:

$$
\begin{aligned}
E^{c v}\left(c_{1}, c_{2}\right)= & \mu \cdot \text { Length }(C)+v \cdot \operatorname{Area}(\text { inside }(C)) \\
& +\lambda_{1} \int_{\text {inside }(C)}\left|I-c_{1}\right|^{2} d x d y+\lambda_{2} \int_{\text {outside }(C)}\left|I-c_{2}\right|^{2} d x d y
\end{aligned}
$$

Wherein, $c_{1}$ and $c_{2}$ are two sets of data attributes with similar interest preferences, $\operatorname{Length}(C)$ indicate the length of the user's browsing of the web user's data, Area(inside $(C)$ ) is normalized time width representing a data distribution region, according to the semantic correlation between the semantic information of the web 
page and the search term of the user, the fuzzy registration is carried out, and the recommended output is obtained as follows:

$$
\begin{aligned}
& C=\operatorname{Min}\left\{\max \left(C_{i}\right)\right\} \\
& \sum_{j^{\prime}=1}^{p^{\prime}} Z_{i, j, j^{\prime}}=1 \quad \forall i \in(1, n), \forall j \in\left(1, n_{i}\right)
\end{aligned}
$$

Wherein, $C_{i}$ represents the confidence level of the page data access time, $Z_{i, j, j^{\prime}}$ is a discrete sample sequence representing association rules. Set the data stream of personalized recommendation output of web pages with uniform ergodicity. The association rules attributes to be mined are as follows $S=\overline{X_{1}}, \overline{X_{2}}, \cdots, \overline{X_{k}}, \cdots$, association rule attribute sets are distributed over time periods, the mutual information function of the personalized recommendation of the web page is described as:

$$
X_{p}(u)= \begin{cases}p \sqrt{\frac{1-j \cot \alpha}{2 \pi}} \mathrm{e}^{j \frac{u^{2}}{2} \cot a} \int_{-\infty}^{+\infty} x(t) \mathrm{e}^{\frac{j^{2}}{2} \cot \alpha-j u c \csc \alpha} d t, & \alpha \neq n \pi \\ x(u), & \alpha=2 n \pi \\ x(-u), & \alpha=(2 n \pm 1) \pi(24)\end{cases}
$$

In the formula, $p$ is interest calculation function of user behavior, $\alpha$ is the width of the time window of the data sampling is improved according to the analysis and the personalized recommendation algorithm of the web page is realized.

\section{SIMULATION EXPERIMENT AND PERFORMANCE ANALYSIS}

In order to test the application performance of this method in the realization of personalized recommendation of web pages, the simulation experiment is carried out. The simulation environment is Myeclipse 8.0 experimental simulation platform. Java platform is used to develop and design the algorithm. The experimental data come from the large comprehensive website of Slashdot. The crawler crawling method is used to capture the data. The page contains 83,244 users and 512,267 sides of user comment information, which is used as a set of priori rule information recommended by the web page. The other related parameters are: $Q=40, c_{1}=120, c_{2}=350$, $c_{r=26}, \mu_{1}=\mu_{2}=0.131, \rho_{1}=\rho_{2}=0.41, \delta=0.8$. The quality of the personalized recommendation is analyzed with an average absolute error (MAE) and an RMSE, the recommended result response output is shown in Figure 1.

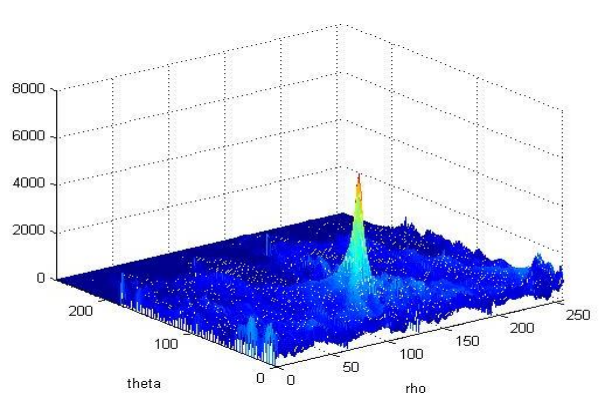

Fig. 1 Web page personalized recommendation response performance distribution

The analysis of Figure 1 shows that this method is used for personalized recommendation of web pages, and the response performance of the output is better, it has strong convergence property. Using different methods to test the recommendation satisfaction estimation dismissal, the root mean square error (RMSE) between the evaluation value and the actual evaluation value is used as the test index, and the result of the comparison between the evaluation values and the actual evaluation value is shown in Figure 2.

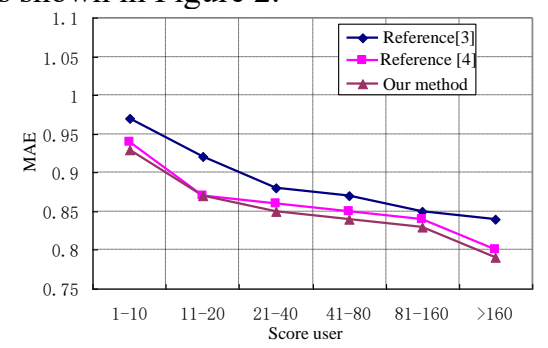

Fig. 2 MAE contrast experimental results

The root mean square error of RMSE personalized recommendation value comparison result is shown in Figure 3.

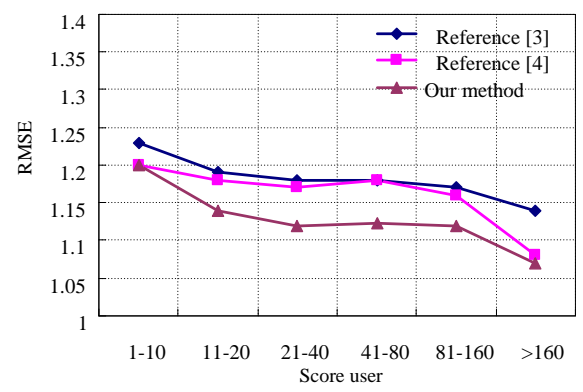

Fig. 3 RMSE contrast experimental results

Comparing the method and the two reference methods, the results are found: The RMSE value of the proposed method is less than the RMSE value of the mean method when $\mathrm{w} 1$ is between $[0.5,1]$. and the RMSE value of the method is less than the RMSE value of the fuzzy $\mathrm{C}$ means recommendation algorithm when $\mathrm{w} 1$ is between $[0.54,0.87]$, that is the recommendation accuracy is higher than that of the fuzzy C - means recommendation algorithm. The combined analysis shows that this method has better accuracy, higher satisfaction and better performance. 


\section{CONCLUSIONS}

In order to improve the efficiency of web page access, it is necessary to establish a personalized recommendation model for web page access to meet the needs of users' personalized interest in web browsing. The personalized recommendation model for web page visit is established to meet the personalized interest demand of the user to browse the web page. A webpage personalized recommendation algorithm based on association rule mining is proposed. Based on the semantic features of web pages, user browsing behavior is calculated by similarity computation, and web crawler algorithm is constructed to extract the semantic features of web pages. The autocorrelation matching method is used to match the features of web page and user browsing behavior, and the association rules feature quantity of user browsing website behavior is mined. According to the semantic relevance and semantic information of web users to search words, fuzzy registration is taken, Web personalized recommendation is obtained to meet the needs of the users browse the web. The simulation results show that the method is accurate and user satisfaction is higher, it has very good application value.

\section{ACKNOWLEDGEMENTS}

This project is supported by and 2017 Undergraduate Scientific and Technological Innovation Project Fund of Guangdong Province (pdjh2017b0943).

\section{REFERENCES}

1. Liu Junyan, Liu Yang, Wang Fei, et al. Study on probability of detection(POD) determination using lock-in thermography for nondestructive inspection(NDI) of CFRP composite materials[J]. Infrared Physics \& Technology, 2015, 71:448-456.
2. Kumar A, Pooja R, and Singh G K. Design and performance of closed form method for cosine modulated filter bank using different windows functions[J]. International Journal of Speech Technology, 2014, 17(4): 427-441.

3. PENG Xiongbin, GONG Guofang, LIAO Xiangping, WU Weiqiang, WANG Hui, LOU Haiyang. Modeling and Model Identification of Micro-position-control Hydraulic System. Journal of Mechanical Engineering, 2017, 53(22): 206-211.

4. MA Zikui, CHEN Wenhua. Friction Torque Calculation Method of Ball Bearings Based on Rolling Creepage Theory. Journal of Mechanical Engineering, 2017, 53(22): 219-224.

5. CHEN W,MA Z,GAO L,et al. Quasi-static analysis of thrust-loaded angular contact ball bearings part I:Theoretical formulation[J]. Chinese Journal of Mechanical Engineering,2012,25(1):71-80.

6. ZHAO Chunjiang,WANG Jianmei,HUANG Qingxue. Modification of raceway control theory on the dynamic equations group of high-speed ball bearings[J]. Transactions of the Chinese Society for Agricultural Machinery,2009,40(5):199-202.

7. SU Bing,ZHANG Shuo,YANG Boyuan. Experiment for sliding friction characteristics of bearing steel GCr15[J]. Bearing,2015(2):39-42.

8. LIN Dejia, ZANG Mengyan. Research on Impact Fracture Behavior of the Laminated Glass Based on Cohesive Zone Model. Journal of Mechanical Engineering, 2017, 53(22): 176-181.

9. CHEN S, ZANG M, XU W. A three-dimensional computational framework for impact fracture analysis of automotive laminated glass[J]. Computer Methods in Applied Mechanics and Engineering, 2015, 294:72-99. 